\title{
Endoscopic Nasobiliary Drainage Help to Prevent the Postoperative Complications of ERCP for Choledocholith Removal
}

\author{
Zhu Qingyun, Pan Xinting*, Wang Yunlong, Liu Fuguo, Sun Yunbo, Li Liandi, Yu Bangxu, \\ Jiang Wenbin, Li Kun, Wang Huimin, Sui Na
}

The Intensive Care Unit of the Affiliated Hospital of Qingdao University, Qingdao, China

Email address:

0536pxt@163.com (Pan Xinting)

${ }^{*}$ Corresponding author

\section{To cite this article:}

Zhu Qingyun, Pan Xinting, Wang Yunlong, Liu Fuguo, Sun Yunbo, Li Liandi, Yu Bangxu, Jiang Wenbin, Li Kun, Wang Huimin, Sui Na. Endoscopic Nasobiliary Drainage Help to Prevent the Postoperative Complications of ERCP for Choledocholith Removal. European Journal of Clinical and Biomedical Sciences. Vol. 2, No. 6, 2016, pp. 83-86. doi: 10.11648/j.ejcbs.20160206.14

Received: August 15, 2016; Accepted: November 17, 2016; Published: December 17, 2016

\begin{abstract}
Aims: In order to investigate the preventive effect of endoscopic nasobiliary drainage (ENBD) on the postoperative complications of ERCP with endoscopic sphincterotomy (EST) for choledocholith. Methods: we collected 180 patients with choledocholith who received the treatment of ERCP for extracting stones between January 2007 and June 2009. All of the 180 patients were divided into two groups: the ENBD group which, including 80 patients, were placed with ENBD, and the control group, including the residual 100 patients, were not placed with ENBD. We measured the scrum amylase levels within 24 hours, before and after ERCP. Meanwhile, the incidences of hyperamylasemia, acute pancreatitis, cholangitis and sphincter of Oddi dysfunction (SOD) were observed after ERCP. Results: The ENBD group shown lower scrum amylase levels after ERCP $(225.78 \pm 178.62$ vs. $563.08 \pm 201.24, \mathrm{P}<0.05)$ than control group, while not significant difference existed between these two groups (130.25 \pm 35.20 vs. $145.35 \pm 45.34, \mathrm{P}>0.05)$ before ERCP. At the same time, ENBD group were found with less incidences of hyperamylasemia ( $5 \%$ vs. $18 \%, \mathrm{P}<0.05)$, acute pancreatitis $(2.5 \%$ vs. $8 \%, \mathrm{P}<0.05)$ and $\mathrm{SOD}(5 \%$ vs. $20 \%, \mathrm{P}<0.05)$, but not different in cholangitis $(2.5 \%$ vs. $3 \%, \mathrm{P}>0.05)$ than control group. Conclusion: Out results suggested that ENBD can prevent the complications of hyperamylasemia, acute pancreatitis and SOD after ERCP effectively.
\end{abstract}

Keywords: Endoscopic Retrograde Cholangiopancreatography, Endoscopic Nasobiliary Drainage, Endoscopic Sphincterotomy, Hyperamylasemia, Acute Pancreatitis, Cholangitis

\section{Introduction}

With the development of ERCP technology, therapeutic ERCP has played an important pole in the treatment of biliary and pancreatic diseases. Especially, the treatment of ERCP with endoscopic sphincterotomy (EST) has a high achievement in curing choledocholith, but it can bring out a series of complications such as hyperamylasemia, acute pancreatitis, hemorrhage and perforation. And some severe complications can lead to death [1-4]. Therefore, it is valuable to investigate the measures to prevent postoperative complications of ERCP for choledocholith. Although some researcher had reported that ENBD was a certain preventive effect on postoperative complications, whether ENBD performed after therapeutic ERCP has not been concluded [1-3]. We had retrospectively analyzed the condition of complications after ERCP for extracting stones, and compared the incidence of complications in cases when naso-biliary tube procedure was performed with in cases was not. To comprehend whether postoperative complications of ERCP for extracting stones are prevented or reduced by ENBD performed.

\section{Materials and Methods}

From January 2007 to June 2009, we collected 180 
patients diagnosed by CT, ultrasonography, MRCP who were admitted to receive ERCP concomitantly with EST for stone removed. According to whether ENBD performed after operation, the patients were divided into two groups. There were not significant differences among them in age, gender, etiology, clinical feature, number and size of stones, endoscopic therapies and clinical medication before and after ERCP. Additionally, patients with acute pancreatitis or cholangitis were excluded before ERCP. The ENBD group contained obstructive jaundice13cases, post-cholecystectomy 17 cases and pancreatic duct visualization procedure 5 cases, meanwhile, the control group contained obstructive jaundice11cases, post- cholecystectomy 24 cases, and pancreatic duct visualization procedure 4 cases.

Equipments: The equipments we used in the procedure as follows: duodenoscopes (Olympus, JF240 and TJF240), high frequency electric deviser (Olympus PSD-20), x-ray machine (OEC-9800), nasobiliary drainage tube (Wilson Cook Co. diameter $7 \mathrm{Fr}$, types of left bend, pigtail and right bend), zebras guide wire, calculus removed basket, lithotripsy basket and calculus removed balloon (Boston Scientific Co.).

Methods:

Procedure: In fifteen minutes before ERCP, patients needed to take Lidocaine Hydrochloride Mucliage at the dose of $10 \mathrm{ml}$. Meanwhile, patients were intramuscularly injected with diazepam at the dose of $10 \mathrm{mg}$, Pethidine $50 \mathrm{mg}$ and 654-2 10mg. The dose of diazepam and Pethidine could be reduced to half, if the patients are over 60 years old. All patients were taken with ECG monitering and Oxygen during operation procedure. Endoscopist operated the duodenoscope reaching to descending duodenum, and found out the opening of nipple. After a pull-type papillotome inserted the bile duct following cholangiography to determine the diagnosis of common bile duct stone. EST was performed in all patients by a pull-type papillotome prior to extracting stones by basket or balloon. If the large stone was difficult to extract directly by basket, basket lithotripsy was carried out first. Finally, cholangiography underwent again to make sure no residual stones left. The naso-biliary tube was inserted into appropriate position of intrahepatic or extrahepatic bile duct by guidwire. Under X-ray, keep the nasobiliary tube in its original position, retreat the duodenoscope gradually from the mouth. The nasobiliary tube was elicited through the nostril, fixed appropriately and contacted with a drainage bag. All patients could not eat about 12 to 24 hours until the scrum amylase levels were normal or near normal level.

Observation parameters: Observe the clinical symptoms (abdominal pain, fever, nausea, vomiting, hematemesis and melena) and laboratory findings (WBC, HB, TBIL, scrum amylase) of all patients, and the amount and color of the bile in drainage bag.
The criteria for the diagnosis of complications in 24 hours after ERCP procedure are as follows [8]: (1) Acute pancreatitis was defined as cases in which serum amylase exceeded three times the normal value in the presence of abdominal pain or nausea /vomiting. (2) Hyperamylasemia was defined as cases in which serum amylase exceeded three times the normal value without the symptom and sign presented of acute pancreatitis. (3) Cholangitis was defined as cases in which in the presence of right upper abdominal pain and fever over 38.5 degree with $\mathrm{WBC}>10 \times 10^{9} / \mathrm{L}$ without other infective disease. (4) Bleeding was defined as cases in which in the presence of hematemesis/melena, or laboratory findings of hemoglobin was lower five percent than the normal value within a day after the procedure. (5) Perforation was defined as cases in which body temperature was high 38.5 degree over two days and with clinical signs of infection and supported by the laboratory findings, there was an image of leakage of gas under diaphragm and behind peritoneum by auxiliary examination. (6) SOD was defined as cases in which in the presence of simple abdominal pain with nausea/vomiting, excepting organic diseases and other complications.

Statistical analyses: Statistical analyses were performed using SPSS for windows, version 11.5. The chi-square test was used for continuous variables expressed as mean \pm standard deviation, and the Fisher exact test for categorical variables. $\mathrm{P}$ values of less than 0.05 were deemed to be statistically significant.

\section{Results}

The serum amylase levels between two groups were viewed by table 1 before and after ERCP. The difference was not statistically significant before ERCP $(\mathrm{P}>0.05)$. However, the mean serum amylase was lower in the ENBD group versus in the control group and the difference was statistically significant after ERCP $(\mathrm{P}<0.05)$. The incidence of hyperamylasemia was higher in the ENBD group versus in the control group $(5 \%$ vs. $18 \%, \mathrm{P}<0.05)$. The incidence of acute pancreatitis was higher in the ENBD group versus in the control group $(2.5 \% \mathrm{vs} .8 \%, \mathrm{P}<0.05)$. The incidence of SOD was higher in the ENBD group versus in the control group $(5 \%$ vs. $20 \%, \mathrm{P}<0.05)$. The incidence of cholangitis was $2.5 \%(2 / 80)$ in the ENBD group similar to $3 \%(3 / 100)$ in the control group. This difference was not statistically significant $(\mathrm{P}>0.05)$. There were no such complications as bleeding, perforation in the two groups. After performed successfully ERCP for choledocholith removal, obstructive jaundice of cases was relived obviously from the laboratory findings and clinical manifestation. Residual stones were found in four cases by nasocholangiography, and underwent the second ERCP for stone removal.

Table 1. Serum amylase of patients in 24 hours before and after ERCP $(X \pm S) U / L$.

\begin{tabular}{llll}
\hline Groups & Number of patients & before ERCP & after ERCP \\
\hline ENBD roup & 80 & $130.25 \pm 35.20$ & $225.78 \pm 178.62 *$ \\
Control group & 100 & $145.35 \pm 45.34$ & $563.08 \pm 201.24$ \\
\hline
\end{tabular}

Compared with the control group $* \mathrm{P}<0.05$ 
Table 2. The complications in patients after ERCP (\%).

\begin{tabular}{llllll}
\hline Groups & Number & Hyperamylasemia & AP & Cholangitis & SOD \\
\hline ENBD group & 80 & $4(5 \%)^{*}$ & $2(2.5 \%)^{*}$ & $2(2.5 \%)$ & $4(5 \%)^{*}$ \\
Control group & 100 & $18(18 \%)$ & $8(8 \%)$ & $3(3 \%)$ & $20(20 \%)$ \\
\hline
\end{tabular}

Compared with the control group $* \mathrm{P}<0.05$

\section{Discussion}

It is generally believed that the main complications after ERCP are hyperamylasemia, acute pancreatitis, cholangitis, bleeding and perforation. Hyperamylasemia and acute pancreatitis occur most frequently after ERCP, among them the incidence of hyperamylasemia can reach up to $52.17 \%$ that has been reported in the literature [9]. At present, the mechanism of the occurrence of hyperamylasemia and acute pancreatitis after ERCP is not yet clear. There are lots of relative factors such as mechanicalness, chemistry, enzyme, microorganisms, patient, operator, and operation process. Some study had found out an independent risk factor for postoperative acute pancreatitis that localized mucosal hyperemia, edema or bleeding due to the openings of nipple and (or) pancreatic duct were subjected to the mechanical injury by inserting catheter over and over again. In addition to, another important risk factor was hydrodynamic changes and acinar injury when pancreatic duct was injected with contrast agents [10]. Cholangitis is another common complication after ERCP. In addition to imaging methods and sterilization is not strict in procedure, the most important factor for cholangitis is the bile duct drainage obstruction or poor [11]. Researchers believe that the placing nasobiliary drainage can alleviate the pressure of postoperative bile duct and pancreatic duct, which is conducive to the normal drainage of bile and pancreatic juice, and thus reduce the contrast agent and bile flowing into the pancreatic duct, and the negative factors caused by sphincter injury and spasm. Therefore, endoscopic nasobiliary drainage can reduce the incidence of acute pancreatitis and cholangitis after operation. Therefore, endoscopic nasobiliary drainage can reduce the incidence of acute pancreatitis and cholangitis after operation. Additionally, hyperamylasemia is a performance of the mild damage of the pancreas after ERCP, of which the serum amylase level is often as a transient increased, and more in 24 hours reduces to normal level [12].

In current study, 180 enrolled patients with choledocholith underwent successfully ERCP with EST for extracting stones, between the ENBD group and the control group, the occurrence of hyperamylasemia were four cases and eighteen cases respectively ( $5 \%$ vs. $18 \%, \mathrm{P}<0.05)$, acute pancreatitis were two cases and eight cases respectively $(2.5 \%$ vs. $8 \%$, $\mathrm{P}<0.05)$, SOD were four cases and twenty cases (5\%vs. $20 \%$, $\mathrm{P}<0.05)$. The incidence of acute pancreatitis, hyperamylasemia and SOD in the ENBD group is lower than in the control group. The symptoms of mere abdominal pain with nausea occurred within 24hours after ERCP in taking stones are still unclear. Some researcher believed that symptoms may be related to bile duct visualization, intubation and EST, which resulted to sphincter of oddi dysfunction, biliary drainage impeded and the pressure of bile duct too high, finally lead to biliary spasm $[15,16]$. After given 654-2 and metoclopramide in the positive symptomatic treatment, mere abdominal pain with nausea was quickly brought under control. We believe that nasobiliary duct plays a supportive role in the sphincter of Oddi, which reduced all causes to edema or spasm in sphincter of Oddi. In addition, nasobiliary duct directly drained bile, reduced pressure in biliary tract duct and pancreatic duct, the pancreaticobiliary reflux, which ensured patency of the pancreatic duct. So it has preventive effects on the occurrence of acute pancreatitis, hyperamylasemia and SOD.

The nasobiliary drainage carried out after ERCP could thoroughly drain bile to alleviate the pressure of the biliary tract and to reduce the incidence of acute cholangitis, specially there was the case of stones not extracted one time and biliary obstruction after operation was particularly applicable to $[7,13,14]$. In current study, as to the occurrence of cholangitis, there were two cases in the ENBD group and three cases in the control group after ERCP $(2.5 \%$ vs. $3 \%, \mathrm{P}>0.05)$. The deference in the incidence of cholangitis is not statistically significant. This result may originate from using equipments and operating procedures under aseptic condition and patients injected intravenously with the preventive use of antibiotics before and after ERCP. However, there are some differences in severity of the inflammation and the length of course. In the ENBD group two patients were mild cholangitis whose symptoms were under control in 2-3 days, while in the control group there was one out of three mild cholangitis patients developed to severe cholangitis under control in 4-5 days. Through the naso-biliary tube to extract the bile for bacterial culture and sensitivity of drug, we can use effectively antibiotics according to culture results while wash the naso-biliary tube using gentamicin with saline every day. It provides the necessary measures to the treatment of cholangitis. It remains to be further studied whether ENBD can prevent the cholangitis after ERCP for exacting stones.

For patients with multiple stones and larger stones through the machine lithotripsy can not get to be extracted one time and a larger stone was difficult to be extracted in which could be placed nasobiliary drainage that had a certain role of softening stone and reimaging [17]. When we performed successfully ERCP for extracting stones following ENBD after the two to four days, naso-biliary tube may be pulled out if there was not residual stone left through naso-cholangiography and excepting for cases with cholangitis. Among them, four cases still had residual stones left followed ERCP again to extract stones.

Our research shows that placing ENBD after ERCP with 
EST for extracting bile duct stones is a positive preventive effect on the occurrence of hyperamylasemia, acute pancreatitis and SOD. Through the nasobiliary tube for Cholangiography can detect residual stones, and obtain the bile specimens for bacterial culture and clean the conduct bile duct. In conclusion, it is a necessary for patients with bile duct stones to conventionally place the nasobiliary duct after receiving ERCP for extracting stones.

\section{References}

[1] Jakobs R, Benz C, Leonhardt A, et al. Pancreatic endoscopic sphincterotomy in patients with chronic pancreatitis: a single-center experience in 171 consecutive patients [J]. Endoscopy, 2002, 34 (21): 551-554.

[2] Cotton PB, Lehman G, Vennes J, et al. Endoscopic sphincterotomy complications and their management: an attempt at consensus [J]. Gastrointest Endosc, 1991, 37 (12): 383-393.

[3] Kozarek RA, Ball TJ, Patterson DJ, et al. Endoscopic pancreatic duct sphincterotomy: indications, technique, and analysis of results [J]. Gastrointest Endosc, 1994, 40 (13): 592-598.

[4] Sherman S, Lehman GA. Endoscopic pancreatic sphincterotomy: techniques and complications [J]. Gastrointest Endosc Clin N Am, 1998, 8 (2): 115-124.

[5] Freeman ML, DiSario JA, Nelson DB, et al. Risk factors for post-ERCP pancreati- tis: a prospective, multicenter study [J]. Gastrointest Endosc, 2001, 54 (4): 425-434.

[6] Masci E, Toti G, Mariani A, et al. Complications of diagnostic and therapeutic ERCP: a prospective multicenter study [J]. Am J Gastroenterol, 2001, 96 (2): 417-423.

[7] Li ZS, Xu GM, Kong ZX, et al. Complications of diagnostic ERCP and treatment [J]. Chinese Journal of Digestive Endoscopy, 2002, 19 (2): 77-81.
[8] Freeman ML, Nelson DB, Sherman S, et al. Complications of endoscopic biliary Sphincterotomy [J]. N Engl J Med, 1996, 33 (5): 909-918.

[9] Tang SJ, Mayo MJ, Rodriguez-Frias E, et al. Safety and utility of ERCP during pregnancy [J]. Gastrointest Endosc, 2009, 69 (3): 453.

[10] Masci E, Toti G, Mariani A, et al. Complications of diagnostic and therapeutic ERCP: a p rospective multicenter study [J]. Am J Gastroenterol, 2001, 9 (6): 417-423.

[11] Mandryka Y, Klimczak J, Duszewski M, et al. Bile duct infections as a late complication after endoscopic sphincterotomy [J]. Pol Merkur Lekarski, 2006, 21 (126): 525.

[12] Wang HJ, Zhan ZG, Yan JF. Clinical analysis of pancreatitis and hyperamylasemia prevented after ERCP following endoscopic nasobiliary drainage $[\mathrm{J}]$. Pancreatic disease science, 2007, 7 (2): 119.

[13] Chen WF, Yao LQ. Postoperative complications of ERCP and EST progress in the prevention and treatment [J]. China Practical Medicine, 2004, 6 (8):66-67.

[14] Loperfido S, Angelini G, Benedetti G, et al. Major early complications from diagnostic and therapeutic ERCP: a prospective multicenter study [J]. Gastrointest Endosc. 1998, 48 (1): 1-10.

[15] Quallich LG, Stern MA, Rich M, et al. Bile duct crystals do not contribute to sphincter of Oddi dysfunction [J]. Gastrointest Endosc 2002, 55 (23): 163-166.

[16] Tzovaras G, Rowlands BJ. Transduodenal sphincteroplasty and transampullary septectomy for sphincter of Oddi dysfunction [J]. Ann R Coll Surg Engl 2002, 8 (4): 14-19.

[17] Xing GP, Wang HY, Yu XP, et al. clinical analysis of 391 cases of common bile duct stones with ERCP, EST and ENBD treatment of [J]. Journal of Clinical Gastroenterology, 2007, 19 (4): 245-246. 